\title{
Associações genéticas entre pesos e características reprodutivas em rebanhos da raça Nelore
}

\author{
Arione Augusti Boligon ${ }^{1}$, Lucia Galvão de Albuquerque ${ }^{2}$, Paulo Roberto Nogara Rorato ${ }^{3}$ \\ ${ }^{1}$ Mestranda em Genética e Melhoramento Animal, FCAV/UNESP - Jaboticabal/SP. Bolsista FAPESP. \\ 2 Departamento de Zootecnia, FCAV/UNESP - Jaboticabal/SP - Pesquisadora do CNPq. \\ ${ }^{3}$ Departamento de Zootecnia, UFSM - Santa Maria/RS.
}

RESUMO - Dados de 14.918 animais da raça Nelore nascidos entre 1991 e 2000, provenientes de rebanhos localizados nas regiões Sul e Sudeste do País, foram utilizados para estimar componentes de co-variância, herdabilidade e correlações genéticas de peso ao desmame (PD), peso a 1 ano de idade (PA), peso ao sobreano (PS), peso ao primeiro parto (PPP), idade ao primeiro parto (IPP) e dias para o primeiro parto (DP). As estimativas dos componentes de co-variância e dos parâmetros genéticos foram obtidas pelo método de máxima verossimilhança restrita, em análises multivariadas. As herdabilidades estimadas para PD, PA, PS, PPP, IPP e DP foram de 0,26; 0,30; 0,34; 0,35; 0,14 e 0,07, respectivamente. Correlações genéticas negativas foram estimadas entre pesos medidos em diferentes idades e IPP, as quais variaram de - 0,31 a $-0,16$. Do mesmo modo, as estimativas de correlação genética entre PD $\times$ DP $(-0,09)$; PA $\times$ DP $(-0,13)$; PS $\times$ DP $(-0,17)$ e PPP $\times$ DP $(-0,16)$ foram negativas, embora de menor magnitude. As correlações genéticas estimadas entre as características de crescimento e a IPP foram favoráveis. Assim, a seleção para aumento de peso deve promover redução da IPP. A alta correlação genética estimada entre IPP e DP $(0,73)$ indica que o uso de DP na seleção de bovinos de corte pode promover resposta correlacionada favorável na idade ao primeiro parto.

Palavras-chave: fertilidade, gado de corte, herdabilidade, parâmetros genéticos

\section{Genetic associations among weights and reproductive traits in Nellore cattle}

\begin{abstract}
Records from 14,918 Nellore animals born from 1991 to 2000 in 28 herds in the South and Southeast regions of Brazil were used to estimate (co)variance components and genetic parameters for weaning weight(W W), yearling weight (YW), weights at 18 month of age (WAY) and at first calving (WFC), age at first calving (AFC) and days to first calving (DC). Multivariate animal models were fitted by REML. Estimates of heritability for WW, YW, WAY, WFC, AFC and DC were 0.26; $0.30 ; 0.34 ; 0.35 ; 0.14$ and 0.07 , respectively. Genetic correlations estimates among growth traits and AFC were moderate and negative, ranging from -0.31 to -0.16 . Genetic correlation estimates were also negative for DC and WW (-0.09); YW $(-0.13)$; WAY (-0.17) and WFC (-0.16). Genetic correlation estimates between growth traits and AFC were favorable and indicate it may be possible to reduce AFC through indirect selection on pos-weaning growth traits. The genetic correlation estimate between AFC and DC was high and positive (0.73) suggesting that selection for DC may result in correlated response for age at first calving.
\end{abstract}

Key Words: beef cattle, fertility, genetic parameters, heritability

\section{Introdução}

Na pecuária de corte, é fundamental selecionar animais visando melhorias nos desempenhos produtivo e reprodutivo, pois esses animais determinam a eficiência total de produção, tanto do ponto de vista genético como econômico. A seleção com base em características de desempenho ponderal tem sido amplamente utilizada, enquanto, entre aquelas indicadoras de fertilidade e precocidade sexual, a única amplamente empregada na maioria dos programas de melhoramento é a circunferência escrotal.

A limitação na utilização de características reprodutivas de fêmeas como critério de seleção ocorre principalmente pela maior dificuldade de mensuração. Além disso, alguns produtores atrasam a entrada de fêmeas na reprodução, determinando idade ou peso para início da vida reprodutiva, o que dificulta a identificação das fêmeas sexualmente precoces.

Um dos maiores desafios entre os produtores é melhorar a eficiência reprodutiva das matrizes de corte, a qual pode

Este artigo foi recebido em 4/6/2007 e aprovado em 28/9/2007.

Correspondências devem ser enviadas para arioneb@bol.com.br. 
ser obtida por meio de seleção para reduzir a idade ao primeiro parto. Entre as principais vantagens em emprenhar novilhas mais jovens, destacam-se o aumento da vida reprodutiva da vaca e do número de bezerros e a redução do tempo para obter retorno do investimento (Short et al., 1994).

A característica dias para o parto tem sido apontada como uma alternativa para avaliar a fertilidade em fêmeas. Segundo Forni \& Albuquerque (2006), esta característica reflete a variabilidade genética das fêmeas em apresentar atividade de estro durante a estação de monta, o número de serviços requeridos para a prenhez, a duração da gestação e a data do parto. Permite ainda a identificação dos animais com maior fertilidade dentro do rebanho, das fêmeas que emprenham mais precocemente dentro da estação de monta e dos touros que produzem filhas que emprenham mais cedo dentro da estação de monta.

O conhecimento das estimativas de correlações genéticas entre características de desempenho ponderal e reprodutivas é fundamental para a inclusão das características reprodutivas nos índices de seleção.Esses resultados permitem predizer, por exemplo, os efeitos da seleção para características como pesos ou ganhos de peso obtidos em diferentes idades sobre o desempenho reprodutivo das matrizes. Entretanto, estudos que relacionam pesos medidos em diferentes idades com características reprodutivas apresentam resultados contraditórios. Mariante \& Zancaner (1985) relataram correlações genéticas positivas (desfavoráveis), de 0,12 a 0,65 , entre pesos medidos em diferentes idades e a idade ao primeiro parto, portanto, os genes responsáveis por maiores pesos seriam também responsáveis por concepções mais tardias em rebanhos da raça Nelore. Por outro lado, correlações genéticas negativas (favoráveis) ou nulas entre pesos da desmama à idade adulta e a idade ao primeiro parto também são descritas na literatura (Mercadante et al., 2000; Silva et al., 2000; Gressler et al., 2005; Pereira et al., 2005).

Em estudo com bovinos de raças Européias, Meyer et al. (1991) relataram correlações genéticas negativas $(-0,10$ a -0,66) entre dias para o parto e características de peso. Em contraste, Johnston \& Bunter (1996) estimaram correlações genéticas positivas e baixas entre dias para o parto e peso à desmama $(0,10)$ e a 1 ano de idade $(0,08)$. No Brasil, Pereira et al. (2001) também relataram correlações genéticas positivas, porém de maior magnitude, entre dias para o parto e pesos ao nascimento e à desmama $(0,48 \mathrm{e}$ 0,28, respectivamente), em rebanhos da raça Nelore. Para essa mesma raça, Forni \& Albuquerque (2005) estimaram correlação genética nula $(-0,02)$ entre dias para o parto e peso aos 550 dias de idade.
Os objetivos neste estudo foram estimar herdabilidades e correlações genéticas entre pesos medidos em diferentes idades (peso à desmama, a 1 ano de idade, ao sobreano e ao primeiro parto) e características reprodutivas (idade ao primeiro parto e dias para o primeiro parto) em rebanhos da raça Nelore.

\section{Material e Métodos}

Foram utilizadas informações de 14.918 animais da raça Nelore, filhos de 811 touros e 9.527 vacas, pertencentes a 28 rebanhos localizados nas regiões Sul e Sudeste do País. O período de observação das características foi de 1991 a 2000.

Consideraram-se somente animais com pai e mãe conhecidos, produtos de inseminação artificial, nascidos de parto simples e criados em regime exclusivo de pastagem. Os grupos de contemporâneos foram definidos de acordo com os efeitos que influenciavam significativamente cada característica, mantendo-se no mínimo cinco animais em cada grupo de contemporâneo. Utilizou-se um arquivo de genealogia contendo identificação do animal, do pai e da mãe, totalizando 27.141 animais na matriz de parentesco.

As características avaliadas (Tabela 1 ) foram peso ao desmame (PD), peso a 1 ano de idade (PA), peso ao sobreano (PS), peso ao primeiro parto (PPP), idade ao primeiro parto (IPP) e dias para o primeiro parto (DP).

As novilhas foram submetidas à reprodução com aproximadamente 27 meses e peso médio de $330 \mathrm{~kg}$. A idade ao primeiro parto, em dias, foi obtida pela diferença entre a data do primeiro parto e a data de nascimento da fêmea. A característica dias para o primeiro parto (DP) correspondeu ao intervalo, em dias, do primeiro dia da estação de monta à data do parto subseqüente.

Foram consideradas duas estações de nascimento, agrupando-se os meses de dezembro a março (estação 1) e

Tabela 1 - Número de observações $(\mathrm{N})$, médias $(\overline{\mathrm{X}})$, desviospadrão (DP), valores mínimo (MIN) e máximo (MAX) e número de grupos de contemporâneos (NGC) para as características avaliadas

\begin{tabular}{lcccccc}
\hline Característica & $\mathrm{N}$ & $\overline{\mathrm{X}}$ & $\mathrm{DP}$ & MIN & MAX & NGC \\
\hline PD (kg) & 14.918 & 178,42 & 28,96 & 131,11 & 201,54 & 451 \\
PA (kg) & 13.342 & 217,29 & 42,58 & 166,99 & 301,79 & 451 \\
PS (kg) & 11.428 & 269,84 & 52,25 & 221,67 & 452,23 & 429 \\
PPP (kg) & 9.932 & 402,93 & 49,31 & 311,06 & 490,07 & 412 \\
IPP (dias) & 9.932 & $1.004,3$ & 111,9 & 723,45 & $1.238,70$ & 386 \\
DP (dias) & 9.932 & 315,21 & 18,91 & 281,32 & 379,90 & 412 \\
\hline
\end{tabular}

PD - peso ao desmame; PA - peso a 1 ano de idade; PS - peso ao sobreano; PPP - peso ao primeiro parto; IPP - idade ao primeiro parto; e DP - dias para o parto. 
junho a setembro (estação 2). A estação de monta foi definida pelos meses de março a maio (estação 1) e outubro a dezembro (estação 2).

Foram realizadas análises de variância, pelo método dos quadrados mínimos, utilizando-se o procedimento GLM (General Linear Models) (SAS, 2001). A partir desse estudo inicial, cujo resultado indicou os efeitos ambientais que estavam influenciando significativamente $(\mathrm{P}<0,05)$ as características a serem avaliadas, foram definidos os grupos de contemporâneos e a co-variável a ser incluída no modelo de avaliação genética.

Os componentes de co-variância foram estimados por máxima verossimilhança restrita não-derivativa utilizando-se o software MTDFREML (Boldman et al., 1995), assumindo o modelo animal. Análises multivariadas são as mais indicadas para avaliações de características medidas após a desmama, uma vez que é possível remover o viés causado pelo efeito da seleção.

Inicialmente, as características foram analisadas por modelos uni e bivariados. Para a estimação das correlações genéticas e fenotípicas entre as variáveis, foram realizadas análises trivariadas. As médias das variâncias e covariâncias genéticas e fenotípicas para cada característica, obtidas nas análises trivariadas, foram usadas para estimação das correlações genética e fenotípica entre as características avaliadas. Em todas as análises bi e trivariadas, foi incluído o peso à seleção (peso à desmama). O critério de convergência considerado foi de $10^{-9} \mathrm{e}$, a cada convergência, o programa foi reiniciado utilizando-se como valores iniciais aqueles obtidos na análise anterior. Na forma matricial, o modelo geral pode ser descrito como:

$$
\mathrm{y}=\mathrm{Xb}+\mathrm{Z}_{1} \mathrm{a}+\mathrm{Z}_{2} \mathrm{~m}+\mathrm{Z}_{3} \mathrm{p}+\mathrm{e}
$$

em que $y=$ vetor de observações; $b=$ vetor dos efeitos fixos; $\mathrm{a}=$ vetor de efeitos genéticos aditivo direto; $\mathrm{m}=$ vetor dos efeitos genéticos aditivo materno; $\mathrm{p}=$ vetor dos efeitos de ambiente permanente materno; e e = vetor dos erros aleatórios residuais associados às observações. $\mathrm{X}, \mathrm{Z}_{1}, \mathrm{Z}_{2}$ e $\mathrm{Z}_{3}$ são matrizes de incidência relacionando b, a, m e p a y. Assumiu-se que $\mathrm{E}[\mathrm{y}]=\mathrm{Xb} ; \operatorname{Var}(\mathrm{a})=\mathrm{A} \otimes \sum_{a}, \operatorname{Var}(\mathrm{m})=$ $\mathrm{A} \otimes \sum_{m}, \operatorname{Var}(\mathrm{p})=\mathrm{I}_{\mathrm{Nm}} \otimes \sum_{p} \mathrm{e} \operatorname{Var}(\mathrm{e})=\mathrm{I}_{\mathrm{N}} \otimes \sum_{e}$, em que $\sum_{a}=$ matriz de co-variâncias genética aditiva direta entre as características; $\sum_{m}=$ matriz de co-variâncias genética aditiva materna; $\sum_{p}=$ matriz de co-variâncias de ambiente permanente materno; $\sum_{e}=$ matriz de co-variâncias residual; $\mathrm{A}=$ matriz de parentesco $\mathrm{I}=$ matriz identidade; $\mathrm{Nm}=$ número de mães dos animais com registros; $\mathrm{N}=$ número de animais com registros; e $\otimes=$ produto direto entre matrizes. Os efeitos genético materno e de ambiente permanente materno não foram incluídos para as características PS, PPP, IPP e
DP. Assumiu-se ainda que os vetores a, $m, p$ e e não são correlacionados entre si.

Os efeitos fixos considerados para cada característica são descritos a seguir:

PD e PA: grupo de contemporâneos (incluindo animais nascidos em mesma fazenda, ano e estação e pertencentes ao mesmo sexo e grupo de manejo à desmama); como co-variáveis, consideraram-se a idade do animal à pesagem e a idade da mãe ao parto (efeitos linear e quadrático);

PS: grupo de contemporâneos (incluindo animais nascidos na mesma fazenda, ano e estação e pertencentes ao mesmo sexo e grupo de manejo à desmama e ao sobreano); como co-variável, considerou-se a idade do animal à pesagem (efeitos linear e quadrático);

PPP: grupo de contemporâneos (animais nascidos em mesma fazenda e ano, pertencentes ao mesmo grupo de manejo ao sobreano, estação de monta e ano de parição); como co-variável, considerou-se a idade da vaca ao parto (efeitos linear e quadrático);

IPP: grupo de contemporâneos (animais nascidos em mesma fazenda e ano, pertencentes ao mesmo grupo de manejo ao sobreano e estação de monta);

DP: grupo de contemporâneos (animais nascidos em mesma fazenda e ano, pertencentes ao mesmo grupo de manejo ao sobreano, estação de monta e ano de parição) e sexo do bezerro; como co-variável, considerou-se a idade da vaca ao parto (efeitos linear e quadrático).

\section{Resultados e Discussão}

As magnitudes das estimativas de herdabilidade do efeito genético direto obtidas para PD, PA e PS (Tabela 2) foram próxima aos valores descritos na literatura para bovinos de raças zebuínas, que variam de 0,16 a 0,$37 ; 0,21$ a 0,32; e 0,15 a 0,35, respectivamente (Cabrera et al., 2001; Pereira et al., 2001; Ferraz Filho et al., 2002; Talhari et al., 2003; Forni \& Albuquerque, 2005; Pereira et al., 2005). Do mesmo modo, Albuquerque \& Meyer (2001) e Dias et al. (2005) relataram redução na estimativa de herdabilidade para pesos do nascimento até próximo ao desmame e aumento após este período até o sobreano. Os errospadrão das estimativas de herdabilidade obtidos em análises univariadas foram baixos, com valores de 0,$03 ; 0,03$; 0,04; 0,07; 0,01 e 0,07 para PD, PA, PS, PPP, IPP e DP, respectivamente.

As estimativas de herdabilidade do efeito genético materno para PD e PA estão de acordo com a amplitude descrita na literatura, ou seja, 0,08 a 0,17 e 0,08 a 0,16, respectivamente (Mercadante et al., 2000; Cabrera et al., 
Tabela 2 - Estimativas de co-variância e parâmetros genéticos para características de crescimento e reprodução em análises bivariadas com peso à desmama

\begin{tabular}{lcccccc}
\hline Estimativa & PD & PA & PS & PPP & IPP & DP \\
\hline$\sigma^{2}{ }^{\mathrm{a}}$ & 203,81 & 293,68 & 348,92 & 421,20 & 394,63 & 128,74 \\
$\sigma^{2}{ }^{\mathrm{m}}$ & 79,92 & 50,24 & - & - & - & - \\
$\sigma^{2}{ }_{\mathrm{c}}$ & 74,43 & 48,69 & - & - & - & - \\
$\sigma_{\mathrm{am}}$ & $-31,62$ & $-26,39$ & - & - & - & - \\
$\sigma^{2}{ }_{\mathrm{e}}$ & 422,28 & 585,41 & 682,26 & 776,07 & $2.446,58$ & $1.723,29$ \\
$\mathrm{~h}^{2}{ }^{\mathrm{a}}$ & 0,26 & 0,30 & 0,34 & 0,35 & 0,14 & 0,07 \\
$\mathrm{~h}^{2}{ }^{\mathrm{a}}$ & 0,10 & 0,08 & - & - & - & - \\
$\mathrm{r}_{\mathrm{am}}$ & $-0,27$ & $-0,49$ & - & - & - & - \\
\hline
\end{tabular}

PD - peso ao desmame; PA - peso a um ano de idade; PS - peso ao sobreano PPP - peso ao primeiro parto; IPP - idade ao primeiro parto; DP - dias para o parto; $\sigma^{2}$ - variância genética aditiva direta; $\sigma_{m}^{2}$ - variância genética aditiva materna; $\sigma_{c}^{2}$ - variância atribuída ao efeito de ambiente permanente materno; $\sigma_{\mathrm{am}}$ - co-variância genética entre os efeitos direto e materno; $\sigma^{2}$ - variância ambiental; $h^{2}$ - herdabilidade do efeito direto; $h^{2}{ }_{m}$ herdabilidade do efeito materno; $r_{a m}$ - correlação genética entre efeitos direto e materno.

2001; Bertazzo et al., 2004). Segundo Mercadante et al. (2000), são poucos os estudos que incluem o efeito materno para a característica de PA; de qualquer modo, parece lógico que esta característica apresente menores valores para esses componentes, uma vez que a influência dos efeitos maternos sobre os pesos após a desmama é consequência de efeitos em pesos anteriores a estas idades. Ao estimarem efeitos genéticos diretos e maternos para animais da raça Nelore do nascimeto aos 630 dias de idade, Albuquerque \& Meyer (2001) observaram que, em idades após a desmama, apesar de sua relevância diminuir, este efeito continua presente.

Em análises utilizando informações de animais da raça Canchim, Silva et al. (2000) estimaram valor de herdabilidade inferior $(0,33)$ para a característica PPP em relação à obtida neste estudo. Por outro lado, estimativas de herdabilidade superiores foram encontradas por Talhari et al. (2003) e variaram de 0,37 a 0,42 em análises bivariadas para raça Canchim.

Em geral, as estimativas de herdabilidade obtidas para pesos em diferentes idades neste estudo foram de magnitude moderada, indicando que, parte considerável da variação entre os animais, para estas características, é decorrente das diferenças no mérito genético dos animais; desse modo, os pesos da desmama ao primeiro parto podem responder de forma eficiente à seleção individual.

A herdabilidade estimada para IPP (Tabela 2) condiz com a maioria dos valores descritos na literatura para raças zebuínas, que variam de 0,12 a 0,18 (Silva et al., 2000; Pereira et al., 2001; Talhari et al., 2003; Dias et al., 2004; Boligon et al., 2007). A herdabilidade estimada neste estudo $(0,14)$ indica pequena variabilidade genética aditiva na expressão da IPP e sugere que a seleção direta para esta característica deve apresentar pequenas taxas de ganho genético anual. No entanto, considerando a importância econômica das características reprodutivas, os processos seletivos a longo prazo poderão ter impacto positivo na produtividade dos rebanhos.

Ressalta-se que características reprodutivas registradas a campo, como idade ao primeiro parto, são mensuradas apenas em fêmeas consideradas férteis. Assim, as amostras são usualmente viesadas, o que pode contribuir para mascarar a variabilidade real da característica e as diferenças genéticas entre os animais. Apesar de a estação de monta ser essencial para avaliação genética, a utilização de estação de monta de curta duração nos rebanhos estudados pode ter contribuído para que a estimativa de herdabilidade para IPP fosse de baixa magnitude.

Para a característica DP, herdabilidades similares à estimada neste estudo também foram relatadas por Pereira et al. (2002), Mercadante et al. (2003) e Forni \& Albuquerque (2005), que variaram de 0,04 a 0,07. Segundo Forni \& Albuquerque (2005), baixa estimativa de herdabilidade para DP também pode ser reflexo da utilização nas análises de apenas fêmeas que emprenharam. Assim, mesmo utilizando um arquivo de genealogia completo, muitas vezes não é possível obter estimativa acurada da variabilidade genética na população.

As correlações genéticas estimadas entre as características ponderais apresentaram valores positivos e altos, de modo que maiores estimativas de correlações genéticas foram obtidas entre pesos adjacentes, reduzindo as estimativas à medida que as idades se distanciam. Esses resultados indicam que a seleção para o aumento de peso em qualquer idade promoverá mudanças na mesma direção nos demais pesos. As estimativas obtidas neste estudo corroboram as descritas por Ferraz Filho et al. (2002), Malhado et al. (2002) e Santos et al. (2005), que variaram de 0,75 a $0,90(\mathrm{PD} \times \mathrm{PA}) ; 0,61$ a $0,83(\mathrm{PD} \times \mathrm{PS})$ e 0,65 e $0,97(\mathrm{PA} \times \mathrm{PS})$ para as raças Tabapuã, Guzerá e Nelore, respectivamente. Por outro lado, Mucari et al. (2003) relataram correlações genéticas inferiores entre peso aos 8 meses de idade e PA $(0,73)$ e PA $\times$ PS $(0,62)$ em rebanhos da raça Canchim.

As correlações genéticas estimadas entre PPP e demais pesos foram superiores às relatadas por Talhari et al. (2003), que estimaram valores de 0,66 $(\mathrm{PPP} \times \mathrm{PD}), 0,77(\mathrm{PPP} \times \mathrm{PA})$ e 0,65 (PPP $\times$ PS $)$ para fêmeas da raça Canchim. A utilização do peso como critério de seleção é fundamental, uma vez que os produtores são remunerados pelo peso vivo dos animais ao abate. Entretanto, os resultados sugerem que a seleção para maiores pesos em idades jovens acarreta, por resposta correlacionada, maiores pesos em idades mais 
Tabela 3 - Correlações genéticas (acima da diagonal) e fenotípicas (abaixo da diagonal) entre as características de crescimento e reprodução em análises trivariadas incluindo o peso à desmama em todas as análises

\begin{tabular}{lcccccc}
\hline & PD & PA & PS & PPP & IPP & DP \\
\hline PD & - & 0,84 & 0,81 & 0,79 & $-0,16$ & $-0,09$ \\
PA & 0,69 & - & 0,79 & 0,83 & $-0,23$ & $-0,13$ \\
PS & 0,64 & 0,66 & - & 0,86 & $-0,29$ & $-0,17$ \\
PPP & 0,56 & 0,69 & 0,72 & - & $-0,31$ & $-0,16$ \\
IPP & $-0,18$ & $-0,25$ & $-0,34$ & $-0,49$ & - & 0,73 \\
DP & 0,03 & $-0,10$ & $-0,29$ & $-0,27$ & 0,65 & - \\
\hline
\end{tabular}

PD - peso ao desmame (kg); PA - peso a 1 ano de idade, $\mathrm{kg}$; PS - peso ao sobreano $(\mathrm{kg})$; PPP - peso ao primeiro parto $(\mathrm{kg})$; IPP - idade ao primeiro parto (dias); e DP - dias para o parto.

avançadas e tem como conseqüência direta maiores requerimentos nutricionais para mantença das matrizes.

As estimativas de correlações genéticas entre as características ponderais e a IPP foram baixas a moderadas e de sentido favorável à seleção. Correlações genéticas negativas entre pesos medidos em diferentes idades e a IPP também foram descritas por Mercadante et al. (2000) e Talhari et al. (2003) para as raças Nelore e Canchim, respectivamente. Esses resultados caracterizam ação de sinergismo entre genes favoráveis às características ponderais e aqueles ligados à expressão da idade ao primeiro parto, ou seja, fêmeas com maior potencial de crescimento pós-desmama tendem a ser mais precoces ao primeiro parto.

A correlação genética estimada entre IPP e DP indica a possibilidade de obtenção de resposta correlacionada favorável em termos de redução da idade ao primeiro parto usando DP como critério de seleção. Entretanto, esta resposta deve ser lenta, uma vez que a variação genética para esta característica não é grande. Resultado semelhante foi relatado por Forni \& Albuquerque (2005), que estimaram correlação genética de 0,94 entre IPP e DP. Simioni (2002) também relatou correlação genética favorável entre IPP e data do parto $(0,39)$ e concluiu que a seleção simultânea para estas características pode ser um método eficaz para obtenção de ganhos genéticos em precocidade sexual e fertilidade.

Em geral, os resultados deste estudo indicam associações entre as características de crescimento e reprodutivas no sentido desejável, entretanto, as correlações são apenas moderadas, indicando resposta lenta na redução da IPP quando considerado o desempenho ponderal em programas de melhoramento. Assim, a inclusão de características reprodutivas nos índices de seleção pode ser uma boa alternativa para melhorar, de forma mais rápida, a fertilidade e precocidade sexual nos rebanhos estudados.

\section{Conclusões}

A seleção com base nas características idade ao primeiro parto e dias para o parto pode melhorar o desempenho reprodutivo de fêmeas, entretanto, a resposta à seleção individual deve ser pequena. A utilização de dias para parto como critério de seleção pode promover resposta correlacionada favorável na idade à primeira concepção e contribuir na obtenção de ganhos em precocidade sexual de fêmeas. Nas condições deste estudo, o descarte de vacas jovens com base no desempenho ponderal após a desmama pode reduzir, a longo prazo, o tempo para entrada em reprodução, o que é desejável.

\section{Literatura Citada}

ALBUQUERQUE, L.G.; MEYER, K. Estimates of direct and maternal genetic effects for weights from birth to 600 days of age in Nelore cattle. Journal of Animal Breeding and Genetics, v.118, p.83-92, 2001.

BERTAZZO, R.P.; FREITAS, R.T.F.; GONÇALVES, T.M. et al. Parâmetros genéticos de longevidade e produtividade de fêmeas da raça Nelore. Revista Brasileira de Zootecnia, v.33, n.5, p.1118-1127, 2004.

BOLDMAN, K.G.; KRIESE, L.A.; Van VLECK, L.D. et al. A manual for use of MTDFREML: a set of programs to obtain estimates of variance and (co)variance (DRAFT). Lincoln: Department of Agriculture/ARS, 1995. 120p.

BOLIGON, A.A.; RORATO, P.R.N.; ALBUQUERQUE, L.G. Correlações genéticas entre medidas de perímetro escrotal e características produtivas e reprodutivas de fêmeas da raça Nelore. Revista Brasileira de Zootecnia, v.36, n.3, p.565571, 2007.

CABRERA, M.E.; GARNERO, A.V.; LÔBO, R.B. et al. Efecto de la incorporación de la covarianza genética directa-materna em el análisis de características de crecimiento em la raza Nelore. Livestock Research for Rural Development, v.13, n.3, 2001.

DIAS, L.T.; EL FARO, L.; ALBUQUERQUE, L.G. Estimativas de herdabilidade para idade ao primeiro parto de novilhas da raça Nelore. Revista Brasileira de Zootecnia, v.33, n.1, p.97$102,2004$.

DIAS, L.T.; ALBUQUERQUE, L.G.; TONHATI, H. et al. Estimação de parâmetros genéticos para peso em diferentes idades para animais da raça Tabapuã. Revista Brasileira de Zootecnia, v.34, n.6, p.1914-1919, 2005.

FERRAZ FILHO, P.B.; RAMOS, A.A.; SILVA, L.O.C. et al. Herdabilidade e correlações genéticas, fenotípicas e ambientais para pesos em diferentes idades de bovinos da raça Tabapuã. Archives of Veterinary Science, v.7, n.1, p.65-69, 2002.

FORNI, S.; ALBUQUERQUE, L.G. Estimates of genetics correlations between days to calving and reproductive and weight traits in Nelore cattle. Journal of Animal Science, v.83, p.1511-1515, 2005.

FORNI, S.; ALBUQUERQUE, L.G. Avaliação de fatores de ambiente e estimativas de parâmetros genéticos para a característica dias para o parto na raça Nelore. Revista Brasileira de Zootecnia, v.35, n.4, p.1239-1335, 2006.

GRESSLER, M.G.M.; PEREIRA, J.C.C.; BERGMANN, J.A.G. et al. Aspectos genéticos do peso a desmama e de algumas características reprodutivas de fêmeas Nelore. Arquivo Brasileiro de Medicina Veterinária e Zootecnia, v.57, n.4, p.533-538, 2005 
JOHNSTON, D.J.; BUNTER, K.L. Days to calving in Angus cattle: genetic and environmental effects, and covariances with other traits. Livestock Production Science, v.45, n.1, p.13-22, 1996.

MALHADO, C.H.M.; SOUZA, J.C.; SILVA, L.O.C. et al. Correlações genéticas, fenotípicas e de ambiente entre os pesos de várias idades em bovinos da raça Guzerá no estado de São Paulo. Archives of Veterinary Science, v.7, n.1, p.71-75, 2002.

MARIANTE, A.S.; ZANCANER. A. Crescimento e reprodução em gado Nelore: visão do criador e do pesquisador. São Paulo: Criadores, 1985. 152p.

MERCADANTE, M.E.Z.; LÔBO, R.B.; OLIVEIRA, H.N. Estimativas de (co)variância entre características de reprodução e de crescimento em fêmeas de um rebanho Nelore. Revista Brasileira de Zootecnia, v.29, n.4, p.997-1004, 2000.

MERCADANTE, M.E.Z.; PACKER, I.U.; RAZOOK, A.G. et al. Direct and correlated responses to selection for yearling weight on reprodutive performance of Nelore cows. Journal of Animal Science, v.81, p.376-384, 2003.

MEYER, K.; HAMMOND, K.; MACKINNON, M.J. et al. Estimates of covariances between reproduction and growth in Australian beef cattle. Journal of Animal Science, v.69, p.3533-3543, 1991.

MUCARI, T.B.; OLIVEIRA, J.A. Análise genético-quantitativa de pesos aos 8, 12, 18 e 24 meses de idade em um rebanho da raça Guzerá. Revista Brasileira de Zootecnia, v.32, n.6, p.16041613, 2003.

PEREIRA, E.; ELER, J.P.; FERRAZ, J.B.S. Análise genética de algumas características reprodutivas e suas correlações com o desempenho ponderal na raça Nelore. Arquivo Brasileiro de Medicina Veterinária e Zootecnia, v.53, n.6, p.720-727, 2001.
PEREIRA, E.; ELER, J.P.; FERRAZ, J.B.S. et al. Análise genética de características reprodutivas na raça Nelore. Pesquisa Agropecuária Brasileira, v.37, n.5, p.703-708, 2002.

PEREIRA, J.C.C.; RIBEIRO, S.H.A.; SILVA, M.A. Análise genética de características ponderais e reprodutivas de fêmeas bovinas Tabapuã. Arquivo Brasileiro de Medicina Veterinária e Zootecnia, v.57, p.231-236, 2005 (supl. 2).

SANTOS, P.F.; MALHADO, C.H.M.M.; CARNEIRO, P.L.S. et al. Correlação genética, fenotípica e ambiental em características de crescimento de bovinos da raça Nelore variedade mocha. Archives of Veterinary Science, v.10, n.2, p.55-60, 2005.

SHORT, R.Y.; STAIMILLER, R.B.; BELLOWS, R.L. et al. Breeding heifers at one year of age: biological and economic considerations. In: FIELDS, M.J.; SAND, R.S. (Eds.) Factors affecting calf crop. London: CRC Press, 1994. p.55-68.

SILVA, A.M.; ALENCAR, M.M.; FREITAS, A.R. et al. Herdabilidades e correlações genéticas para peso e perímetro escrotal de machos e características reprodutivas e de crescimento de fêmeas, na raça Canchim. Revista Brasileira de Zootecnia, v.29, n.6, p.2223-2230, 2000 (supl. 2).

SIMIONI, V.M. Estudo genético-quantitativo de algumas características reprodutivas na raça Nelore. Jaboticabal: Universidade Estadual Paulista, 2002. Tese (Doutorado em Zootecnia) - Universidade Estadual Paulista, 2002.

STATISTICAL ANALYSIS SYSTEMS - SAS.User's guide. Version 6.11 ed. Cary: SAS Institute, 2001. (CD-ROM).

TALHARI, F.M.; ALENCAR, M.M.; MASCIOLI, A.S. Correlações genéticas entre características produtivas de fêmeas em um rebanho da raça Canchim. Revista Brasileira de Zootecnia, v.32, n.4, p.880-886, 2003. 